DOI: https://doi.org/10.46296/yc.v5i8edespen.0105

\title{
ESTRATEGIAS GERENCIALES DESDE UNA IDENTIDAD DIFERENCIADORA. UN ACERCAMIENTO DESDE LA VISIÓN FINANCIERA ECUATORIANA.
}

\section{MANAGEMENT STRATEGIES FROM A DIFFERENTIATING IDENTITY. AN APPROACH FROM THE ECUADORIAN FINANCIAL VISION.}

\author{
Zambrano-Velásquez Karen Yulen ${ }^{1 *}$; Vegas-Meléndez Hilarión José ${ }^{2}$ \\ ${ }^{1}$ Economista, Pontificia Universidad Católica del Ecuador, Sede Manabí. Portoviejo, \\ Ecuador. Correo: kzambrano1616@pucesm.edu.ec. ORCID ID: https://orcid.org/0000- \\ 0003-0068-5487 \\ ${ }^{2}$ Docente e investigador de pre y posgrado de la Pontificia Universidad Católica del \\ Ecuador, sede Manabí. Portoviejo - Ecuador. Correo: hvegas@pucesm.edu.ec. \\ ORCID ID: https://orcid.org/000-0002-8526-2979
}

\begin{abstract}
Resumen
La investigación tiene como objetivo principal contextualizar las estrategias diferenciadoras de la gerencia ecuatoriana vinculada a las instituciones financieras, soportada en un enfoque cualitativo e inductivo, con un nivel descriptivo, documental y de campo; para el levantamiento de la información se utilizó la observación directa y un cuestionario en la que se encontraban las preguntas de la entrevista en profundidad; esta entrevista se aplicó a gerentes de alto rango gerencial, como lo es el Presidente de ASOBANCA, así como destacados gerentes de instituciones de alto prestigio bancario en la Provincia de Manabí; para el análisis de los resultados se utilizó la estrategia propuesta por la Teoría Fundamentada en relación a la triangulación de los "códigos in vivo", construcción de los datos a través de un proceso de categorización, obteniendo un grupo de descriptores estratégicos y gerenciales como aporte de esta investigación. Entre las conclusiones más relevantes se encuentra el hecho de que el conocimiento y la experiencia de los usuarios o clientes representa una novedosa dimensión para la construcción de una identidad diferenciadora.
\end{abstract}

Palabras clave: Estrategias, Diferenciación, Gerencia, Financieros.

\begin{abstract}
The main objective of the research is to contextualize the differentiating strategies of Ecuadorian management linked to financial institutions, supported by a qualitative and inductive approach, with a descriptive, documentary and field level; Direct observation and a questionnaire containing the questions of the in-depth interview were used to collect the information. This interview was applied to high-ranking managers, such as the President of ASOBANCA, as well as prominent managers of highly prestigious banking institutions in the Province of Manabí; For the analysis of the results, the strategy proposed by the Grounded Theory in relation to the triangulation of the "in vivo codes" was used, data construction through a categorization process, obtaining a group of strategic and managerial descriptors as a contribution of this research. Among the most relevant conclusions is the fact that the knowledge and experience of users or clients represents a new dimension for the construction of a differentiating identity.
\end{abstract}

Keywords: Strategies, Differentiation, Management, Financial.

Información del manuscrito:

Fecha de recepción: 16 de noviembre de 2020.

Fecha de aceptación: 08 de enero de 2021.

Fecha de publicación: 29 de enero de 2021. 


\section{Introducción}

Las estrategias se pueden considerar como la guía necesaria que tienen los gerentes 0 las organizaciones para poder alcanzar los objetivos y metas propuestas. Si su aplicación es correcta, no solo fue bien diseñada, sino que también permitirá posicionarse en las condiciones requeridas. El termino estrategia tiene un sin número de definiciones y connotaciones, dependiendo de su contexto, ámbito, área 0 simplemente campo de batalla.

Justamente es en el campo de la batalla es donde se generaron las principales estrategias para derrotar al adversario, y como bien se puede asociar la participación de las empresas en los mercados, estos mercados también se convierten en un escenario de batallas, no solo para ganar espacio en el mismo, sino en ocasiones para acabar con la competencia. En su libro el Arte de la Guerra, Sun Tzu (2500 AC), quien ha sido denominado el precursor de la estrategia suprema, señala la importancia de conocerse a sí mismo, al enemigo y mejor aún al campo de batalla. Esos tres componentes permiten visualizar el futuro resultado de la acción presente.

Lógicamente hay quienes han abordado el tema de la estrategia desde diferentes vertientes, por ejemplo, para David (2013) son "los medios por los cuales se lograrán los objetivos" (p.12). Por su parte, Mintzberg y Quinn (2015) la definen como "el patrón o plan que integra las principales metas y políticas de una organización, y a la vez establece, la secuencia coherente de las acciones a realizar" (p.32). A su vez, Mintzberg, Ahlstrand y Lampell (2016) extienden el hecho de que la estrategia es "el patrón de una serie de acciones que ocurren en el tiempo, enfatiza la acción en donde la empresa tendría una estrategia aun cuando no tuviera planes definidos, incluso aunque nadie en la empresa dedique tiempo a establecer objetivos formales" (p.16). Tal como se observa, el establecer un patrón de comportamiento ayuda al alcance de los planes establecidos por la organización empresarial a través de una línea de tiempo focalizada en el mercado. 
No cabe la menor duda de que las estrategias representan la herramienta ideal para todo gerente que bien se considere un estratega exitoso. Claro está que el solo diseño no es suficiente, se requiere precisión quirúrgica al momento de la aplicación y en el tiempo adecuado para su ejecución o puesta en marcha. El gerente estratega debe de ser creativo, hábil con el manejo de las ideas y por demás con una visión futurista; con estas condiciones se puede enfrentar a un mercado cada vez más complejo por la capacidad competitiva e innovadora de las empresas que bien buscan mantener - alcanzar más espacio en un mercado común.

La estrategia no puede ser tratada de una manera simple, esta amerita elementos diferenciadores que conlleven a obtener ventajas en el mercado frente a los competidores; para ello, es necesario configurar una estructura organizacional sustentada en las estrategias gerenciales asociadas a las áreas de competencias; en este sentido Mintzberg (2013) las define como "un proceso tanto visionario como de aprendizaje, pero la visión es inaccesible para aquellos que no pueden ver con sus propios ojos" (p.11); por otro lado, Cedeño V. A; Asencio C., y Villegas A, M. (2019) manifiestan que las estrategias gerenciales "son parte elemental para el cumplimiento progresivo de los negocios, porque la responsabilidad es parte vital para la duración en los mercados mucho más en épocas que estamos en globalización y competitividad" (p.191); estas definiciones intentan establecer una relación directa entre lo visionario y competitivo, siendo una forma de visualizar los próximos escenarios en el contexto en el que se desenvuelven las empresas.

La gerencia estratégica tiene una responsabilidad determinante en la elaboración de ese nuevo escenario y no es más que a través del conocimiento de sus propias capacidades, el reconocimiento de las habilidades de sus competidores y de la construcción de los espacios adecuados dentro del mercado para tener ventaja. Para esto último, la empresa debe impulsar procesos diferenciadores disruptivos en el propio mercado que bien le permitan sacar ventajas sobre sus competidores. 
La investigación tiene como propósito apuntar hacia lo expresado en líneas anteriores, en la que se busca mostrar las estrategias gerenciales aplicadas en las instituciones financieras que bien le permitan determinar sus rasgos diferenciadores en el mercado de la banca, en la se pretende develar las características únicas y distintivas en los servicios que se prestan desde las instituciones financieras ecuatorianas, específicamente desde la banca pública. Para ello, la investigación se plantea la siguiente interrogante: ¿Qué estrategias gerenciales deben aplicar los gerentes ecuatorianos en las instituciones financieras para garantizar un proceso diferenciador en los servicios prestados?

Con el objeto de dar respuesta a esta interrogante, se establece como objetivo principal el contextualizar las estrategias diferenciadoras de la gerencia ecuatoriana vinculada a las instituciones financieras; por otro lado, reflexionar sobre la innovación estratégica basada en la diferenciación en las instituciones financieras; por último, identificar las estrategias diferenciadoras más idóneas para las instituciones financieras vinculadas a la banca pública.

\section{Significados de gerencia de la diferenciación}

Antes de ahondar en la temática de la diferenciación es importante destacar los principales exponentes sobre el término estrategia; en este caso tenemos a Miles y Snow (1978) "establecen las estrategias relacionando las empresas con la capacidad de adaptación al entorno y su orientación estratégica, así como el objetivo que se persiga". Para Johnson y Scholes (1993), "la estrategia es la dirección y el alcance de una organización a largo plazo; consigue ventajas para la organización a través de su configuración de los recursos en un entorno cambiante, para hacer frente a las necesidades de los mercados y cumplir las expectativas de los accionistas". Por su parte, Chandler (2003), "la estrategia es la determinación de las metas y objetivos de una empresa a largo plazo, las acciones a emprender y la asignación de recursos necesarios para el logro de dichas metas"; a su vez Ansoff (1965) afirma "que la estrategia es el lazo común entre las 
actividades de la organización y las relaciones producto-mercado de tal manera que definan la esencial naturaleza de los negocios en que está la organización y los negocios que planea para el futuro", posturas interesantes son las Learned, Christensen, Andrews y Guth (1969) quienes dicen de la estrategia "es el patrón de objetivos, propósitos 0 metas, políticas y planes esenciales para lograrlos, establecidos de tal manera que definan en qué clase de negocio está la empresa o quiere estar y qué clase de empresa es 0 quiere ser"; por último, encontramos el aporte de Smith (1977), "la estrategia es la fórmula para obtener éxito en el mundo de los negocios. Es el plan para conseguir los mejores resultados de los recursos, la selección del tipo de negocio en que comprometerse y el plan para conseguir una posición favorable en el campo empresarial".

Al tener una visión más clara sobre las estrategias, se puede avanzar en el término de la diferenciación, para ello se presentan sus principales postulantes comenzando por Porter(1987) propone la estrategia de diferenciación la cual "consiste en generar productos y servicios diferentes para diferentes grupos 0 segmentos de mercado que valoren de manera significativa dichas características diferentes y por ende estén dispuestos a pagar un precio adicional", en este sentido las organizaciones empresariales deben procurar establecer las características más aspiradas por los clientes o usuarios de un mercado, resaltando significativamente un valor por el cual el consumidor considera de exclusividad; sin embargo, el mismo Porter(1987) destaca que "la exclusividad no es una diferenciación a menos que sea valiosa para el cliente". El mismo Porter dentro de sus 3 estrategias genéricas y dentro de las estrategias de diferenciación señala algunos aspectos en los que se puede lograr diferenciación: en el diseño del producto, en sus atributos 0 características, en su desempeño o rendimiento, en la calidad, en la marca, en brindar un buen servicio o atención al cliente en la atención personalizada, en la rapidez, en la entrega, en ofrecer servicios adicionales.

Adicionalmente Franco.C \& Reyes. A (2003) manifiestan que se entiende por diferenciación "la acción que 
emprende una empresa con el fin de introducir un conjunto de atributos significativos para distinguir su oferta de la competencia”, por su parte Gómez (2019) “diferenciación es distinguir un producto o servicio del resto de sus competidores, buscando hacerlo más atractivo a los ojos de su mercado objetivo para que lo prefieran", además Narver y Slater (1990) señalan que "las empresas que adoptan una estrategia de diferenciación es previsible que presten una gran atención a las necesidades presentes y futuras de los consumidores con la finalidad de satisfacerlas de forma más adecuada; es necesario que la empresa analice con gran detalle las estrategias adoptadas por sus principales competidores para garantizar una mayor diferenciación entre su oferta y la de la competencia y es importante que adopte una adecuada coordinación interfuncional que le permita sacar un mayor partido a toda esta información"

\section{Diferenciarse desde el contexto} del servicio

La investigación hizo un acercamiento a los responsables de distintas entidades y expertos a fin de poder caracterizar la discusión en cuanto a los objetivos propuestos por parte de los investigadores. En este sentido, se observan posturas que bien contribuyen el poder contextualizar las estrategias diferenciadoras de la gerencia ecuatoriana vinculada a las instituciones financieras.

En relación a los aspectos condicionantes de un proceso diferenciador en la prestación de los servicios financieros en el Ecuador, Prado (2020) indica que "los servicios financieros están regulados y controlados por la superintendencia de bancos, [...] son gratuitos en el Ecuador lo cual deja muy poco espacio para la diferenciación" (p.1), por su parte Valdivieso (2020) indica "en la banca estamos con estrategias innovadoras eficientes sostenibles, pero siempre y cuando enfocándonos al sector social de manera que lo que nosotros damos se adapten a las necesidades de la población de nuestro banco" (p.2), y 
en el mismo contexto Loor (2020) manifiesta que "en el Ecuador el ente regulador tiene parámetros muy bien definidos de lo que puede hacer una institución bancaria, así como de los costos que puede adquirir o puede cobrar por los mismos" (p.3).

En el sector financiero se percibe la toma de decisiones centralizadas y burocráticas por lo cual las estrategias están diseñadas y direccionadas desde los altos mandos directivos lo que, a consideración de los autores, limita el rediseño y contribuciones de parte de la gerencia del mando medio e incluso los aportes de los colaboradores involucrados de los procesos internos, en la banca pública éstas están dirigidas particularmente al mercado, desviando la atención de los grupos de trabajo de la empresa, éstas estrategias suelen cambiar la aplicación de fondo con cada nueva administración lo que bien pudiere ocasionar retrasos en el cumplimiento de planes estratégicas no por el cambio de mando sino por la poca sostenibilidad de las mismas.

En cuanto a los tipos de direccionamiento estratégico que permite dar un acercamiento a la visión del gerente ecuatoriano en relación a las instituciones financieras Prado (2020) subraya que "la diferenciación viene por elementos muy puntuales o ciertas líneas estratégicas que tiene cada banco, en este caso el direccionamiento estratégico va a estar dado por el segmento de crédito en el cual cada banco decide incursionar" (p.1); sin embargo Valdivieso (2020) apunta que "la estratégica nace de la familia es la primera como visión de un gerente ecuatoriano, es importante la familia y es tan importante que desde esa visión nacen las otras visiones con valores para el mercado y para el público, el enfoque nuestro es inclusivo" (p.2); para lo cual Loor (2020) resalta que "toda la estrategia parte del equipo de trabajo, la estrategia se enfoca en el servicio" (p.3).

Los investigadores se permiten aseverar que, el mercado financiero sí está preparado para este tipo de estrategias, conoce las necesidades, tiene personal capacitado y con potencial, tiene liquidez, pero se encuentra limitado por las regulaciones y normativas vigentes en el país además del riesgo, lo que 
interfiere a la hora de querer instituir nuevos costos por servicios, esto a criterio propio no impide la generación de innovación, la tecnología de por sí en esta nueva era es ineludible por lo tanto se vuelve vital asimilar sus cambios y aprovecharla en función de proyectar y planificar actuales y posibles futuros escenarios.

En la revisión de las estrategias gerenciales que deben aplicar los gerentes ecuatorianos en las instituciones financieras para garantizar un proceso diferenciador en los servicios prestados, para Prado (2020) es importante mantener "productos innovadores atender a los clientes en forma adecuada ampliar la bancarización y buscar nuevos clientes, pero al mismo tiempo lo que quiere siempre el banco es digamos, equilibrar esta parte de la innovación, de la velocidad de ampliar el mercado" (p.1); Valdivieso (2020) destaca que "lo principal sería los grupos prioritarios, en productos, llevar productos, servicios, captaciones, fortalecer las competencias personales, la negociación de los productos de los servicios y la prestación que nosotros hacemos" (p.2); Loor (2020) por su parte acentúa que los colaboradores deben "tener ese compromiso con el banco, deben conocer los objetivos del banco, tener pertenencia al banco para que puedan poner sus mayores esfuerzos, satisfacer el requerimiento del cliente para que se sienta satisfecho" (p.3)

Las tendencias de uso actuales ligadas a la comodidad, seguridad, de fácil uso y acceso que generen respuestas rápidas, son de rápida adaptación sobre todo en las últimas generaciones, siempre y cuando se cumpla con la diferenciación ofertada y se genere ese sentido de confianza, el usuario lo irá asimilando como una nueva necesidad que no sabía que tenía, por otra parte, dentro de la banca pública la aplicabilidad de este tipo de estrategias tomaría un ritmo disímil pues el tipo de cliente sobre todo el externo es diferente, posiblemente de generaciones en las que el uso de la tecnología y digitales se va admitiendo de manera paulatina, por ende la tendencia los lleva a buscar atención personalizada y rápida respuesta , en este caso el apalancamiento debe darse en base a la innovación y 
mejora de procesos internos que permitan cubrir esa necesidad de manera oportuna.

Desde las posturas y experiencias que permitan generar un cambio de visión estratégica (filosofía) basada en la diferenciación del servicio financiero, el presidente Prado (2020) resalta la "importancia de la innovación financiera y la experiencia del consumidor para poder crear nuevos productos financieros, ese el primer elemento, el segundo elemento es una visión muy fuerte hacia la inclusión financiera, y por último la necesidad de poder ampliar la bancarización" (p.1); Loor (2020) en este sentido contempla que "bajo un liderazgo adecuado lograr que el equipo de trabajo brinde un servicio óptimo de calidad y en el tiempo que espera un cliente, para poder sentir satisfacción en el servicio recibido" (p.3) agregando la misma Loor (2020) se "debe apuntar a la transformación digital, en el menor tiempo posible las instituciones financieras del Ecuador" (p.3)

No cabe dudas que la gerencia en el sector bancario dado el contexto actual está traspasando muchas barreras que le han permitido ver las problemáticas y oportunidades desde nuevas perspectivas antes invisibles, ha permitido a los gerentes tomar decisiones rápidas basadas en nuevas técnicas y apoyos como por ejemplo búsqueda de nuevas destrezas o la identificación de nuevas herramientas en línea con la actividad financiera, la experiencia sin duda es válida sin embargo es necesario que los gerentes asuman los nuevos desafíos generando sinergia, confianza y solidaridad con todos los miembros del entorno empresarial.

A manera de propuesta en términos de diferenciación resulta imprescindible pensar en la adopción de nuevas herramientas tecnológicas, simplificación de procesos, seguridad en el amplio basto, generación de experiencias positivas para el usuario a partir de directrices estratégicas y éticas, lo mencionado abre la posibilidad de que el usuario sienta la necesidad de pagar por un producto de calidad y diferenciado, en el caso de la banca pública el efecto pudiera verse direccionado en la adopción de nuevos esquemas mentales por parte del usuario, en este caso 
también se prevé la posibilidad de que la banca necesite destinar más recursos y tiempo para investigación y desarrollo, destacando la importancia de elección y diseño de estrategias pero sobre todo, como aplicarlas y sostenerlas en el marco de la viabilidad.

\section{Identidad metodológica}

La investigación se da desde el enfoque cualitativo, con un nivel descriptivo crítico, apoyada en una metodología inductiva, acompañada de una observación analítica, respaldada en una saturación teórica sobre las principales definiciones sobre las estrategias de diferenciación que se dan en servicios cuyos mercados están marcados por la innovación; el acercamiento a la perspectivas gerenciales se da desde la denominada entrevista en profundidad (instrumento cualitativo), en la que se obtiene información primaria que bien permite conocer en primera instancia el pensamiento e ideas relacionadas con la temática expuesta; la entrevista en profundidad ayuda a:
Obtener las concepciones personales de los entrevistados sobre la situación objeto de la investigación en medio de un esfuerzo de inmersión $y$ reinversión con intimidad $y$ familiaridad para sinceramente reconstruir a través de la discursividad el punto de vista (perspectiva) del informante (o actor relevante) en el cual se ubica la investigación y su contexto (Vegas M., 2016, p. 421)

Las ideas resultantes en la entrevista en profundidad corroborarán las premisas expuestas en la introducción de este trabajo investigativo. Además, en la que expertos en la temática expongan sus ideas de manera libre y directa, basadas en interrogantes $y$ conversatorios direccionados por los investigadores.

El método de análisis de los resultados o hallazgos se da bajo las bases del método hermenéutico, en que lo empírico permite formar parte de la solución del problema, siendo en lo particular para la investigación lo que los entrevistados aporten como ideas para una posterior categorización de los descriptores 
gerenciales resultantes basados en

la perspectiva de las estrategias de diferenciación desde el contexto de las instituciones financieras ecuatorianas.

En este sentido, las entrevistas estuvieron focalizadas a gerentes responsables de entidades financieros, entre los que destacan el presidente de ASOBANCA, Economista Julio José Prado, la Licenciada Adriana Valdivieso (BAN ECUADOR B.P) y la Ingeniera María Adelaida Loor, experta en operaciones bancarias (25 años).

Tabla 1. Categorías y Descriptores Emergentes de la Entrevista en Profundidad.

\begin{tabular}{|c|c|c|c|}
\hline Entrevistado & Códigos en vivo & Categorías & Descriptores \\
\hline $\begin{array}{l}\text { AC: Prado Julio } \\
(2020)\end{array}$ & $\begin{array}{c}\text {-Valor agregado al } \\
\text { cliente } \\
\text {-Precio diferenciado } \\
\text { en función del riesgo } \\
\text {-Servicios financieros } \\
\text { gratuitos } \\
\text {-Ampliación de la } \\
\text { banca } \\
\text {-Segmento } \\
\text { especializado del } \\
\text { banco } \\
\text {-Laboratorios de } \\
\text { innovación bancaria } \\
\text {-Competencias sin } \\
\text { restricciones } \\
\text {-Inclusión } \\
\text { financiera }\end{array}$ & $\begin{array}{c}\text {-Mercados } \\
\text { Saturados } \\
\text {-Productos } \\
\text { innovadores } \\
\text {-Direccionamiento } \\
\text { estratégico por } \\
\text { segmentos } \\
\text {-Democratización } \\
\text { de la banca } \\
\text { financiera }\end{array}$ & $\begin{array}{l}\text { - Mercado bancario } \\
\text { inteligente } \\
\text {-Autonomía de } \\
\text { competencias }\end{array}$ \\
\hline $\begin{array}{l}\text { EO: Valdivieso } \\
\text { Adriana (2020) }\end{array}$ & $\begin{array}{c}\text { - Estrategias } \\
\text { innovadoras, } \\
\text { eficientes y } \\
\text { sostenibles } \\
\text {-Sector social } \\
\text {-Valores desde la } \\
\text { familia para el } \\
\text { mercado } \\
\text { - Competencias } \\
\text { personales }\end{array}$ & $\begin{array}{c}\text { - Banca con valores } \\
\text { familiares y sociales } \\
\text {-Fortalecer } \\
\text { los negocios }\end{array}$ & $\begin{array}{l}\text {-Pensar desde los } \\
\text { valores y la } \\
\text { sostenibilidad social } \\
\text {-Estrategias de } \\
\text { negocios sostenibles }\end{array}$ \\
\hline $\begin{array}{l}\text { EO. Loor María } \\
\text { Adelaida (2020) }\end{array}$ & $\begin{array}{c}\text {-Diferenciación } \\
\text { basada en procesos } \\
\text {-Estrategia focalizada } \\
\text { en el servicio } \\
\\
\text {-Comprometidos con } \\
\text { la filosofía } \\
\text { empresarial }\end{array}$ & $\begin{array}{l}\text {-Valor compartido del } \\
\text { significado calidad } \\
\text {-Alianza cliente- } \\
\text { institución }\end{array}$ & $\begin{array}{l}\text {-Compromisos de } \\
\text { mejoramiento } \\
\text { continuo } \\
\text {-Preparados para las } \\
\text { necesidades }\end{array}$ \\
\hline
\end{tabular}

Fuente: Zambrano \& Vegas (2021) a partir de entrevistas en profundidad.

Notas: AC: Actor de cambio; EO: Experto Operativo. 
Los descriptores que se mencionan a continuación son producto de la comprensión e interpretación de los resultados, mostrados en la tabla 1 , los cuales pretenden aportar a una identidad diferenciadora desde las perspectivas de las estrategias gerenciales abordadas, que a su vez buscan un acercamiento desde la visión financiera ecuatoriana (expresada en las entrevistas en profundidad).

\section{- Mercado bancario inteligente.} Representa un espacio donde la tecnología y la innovación pueden aportar mucho al momento de construir espacios inteligentes dentro del mercado financiero. Sin dudas, representará un aporte significativo para quienes entran a competir por segmentos del mercado financiero bancario, dado que procurarán ser competitivos desde su entrada al mercado. Se hace indispensable un direccionamiento estratégico por segmentos, a través de productos innovadores; Evidentemente, nos encontramos frente a un constante cambio de paradigmas en la que se hace conveniente valorar nuevas formas de experimentar, comunicar, relacionarse y compartir las ideas de negocios sostenibles en los mercados financieros.

- Autonomía de competencias. Las organizaciones financieras para realmente ser competitivas deben de poseer un alto grado de autonomía de competencias, lo cual les permitirá proceder según las nuevas tendencias en materia de digitalización de servicios, llegar a un público un poco más aislado de las tecnologías, pero con una alta posibilidad de aprendizaje y adaptación, ayudando en la democratización de la banca financiera. Se hace necesario que las estrategias gerenciales estén formuladas pensando en las habilidades del diseño y en la heurística que se genera del propio mercado.

\section{- Pensar desde los valores y la sostenibilidad \\ social.}

Indudablemente las organizaciones financieras, y específicamente las instituciones bancarias deben de aprender a considerar los valores sociales al momento de pensar en la prestación de sus servicios y los instrumentos que ofrecen a la ciudadanía generando un impacto positivo en su credibilidad. Existe 
una corresponsabilidad entre las organizaciones empresariales (en este caso, las financieras) y la sociedad, en relación al entorno en común; se hace indispensable que existen políticas compartidas que viabilicen un retorno natural $y$ sostenible hacia el medio ambiente. Se debe de pensar en una banca con valores familiares y sociales, para así ser más empático con los clientes.

- Estrategias de negocios sostenibles. Los negocios de la nueva era han tenido una presencia muy marcada en los últimos tiempos lo cual significa que muchos de ellos se han fortalecido; sin embargo, se hace necesario que estén más comprometidos con la producción responsable y el consumo controlado de los insumos, y más aún si estos provienen de fuente naturales. La banca debe hacer un seguimiento de esta situación, al momento de financiar proyectos que se autodenominen sostenibles, asimismo de ser el caso ofrecer direccionamiento dado el caso que se desconozca del tema o para el desarrollo de ideas por parte de los usuarios o clientes, de tal forma que el negocio garantice beneficios suficientes para que sea económicamente razonable y sustentable.

\section{- Compromisos de mejoramiento}

continuo. El compromiso de calidad en el servicio y en los procesos debe ser una norma en toda organización. Muchas son ejemplo de ello, por lo tanto, su espacio en el mercado es muy merecedor; sin embargo, existen organizaciones financieras en las que sus clientes se ven afectados por clonaciones en sus instrumentos, estafas en línea o situaciones en las que pareciera que la seguridad en estas instituciones está afectada; lo cual hace que sus estrategias y políticas de seguridad no se cumplan de manera efectiva. Debe darse un sentido de valor compartido con los usuarios del significado calidad e innovación, ambas variables son preponderantes en un mercado inteligente; la calidad y la innovación tienen una transferencia al mercado o no son calidad e innovación, y para ello se debe construir un mercado con modelos de negocios que aprovechen tal situación con rápida adaptabilidad. 
- Preparados para las necesidades. Las organizaciones inteligentes, pertenecientes a mercados inteligentes, se anticipan a las necesidades del cliente; generan una conducta de satisfacción y respaldo a la solución. Las instituciones financieras deben de hacer un proceso de alianza más transparente, con verdadero sentido de compromiso mutuo entre el cliente y la institución financiera; donde el cliente sienta confianza en el mercado y no específicamente en una institución en particular. Identificar necesidades desatendidas en el mercado a través de los servicios que prestan es el gran desafío; el poder comercializar nuevas tecnologías, mejorar o transformar un mercado requiere de la participación directa de los usuarios. Es importante tener en cuenta que el usuario tiene el poder de decidir, y lo sabe.

\section{Conclusiones}

La investigación concluye su recorrido entendiendo la importancia de mostrar, desde la perspectiva de la visión financiera ecuatoriana, las estrategias gerenciales desde una identidad diferenciadora que bien le permita abordar el futuro mismo del mercado. Importante es recordar los objetivos propuestos al inicio de la investigación, en la que en primera instancia se buscó el contextualizar las estrategias diferenciadoras de la gerencia ecuatoriana vinculada a las instituciones financieras; por otro lado, el reflexionar sobre la innovación estratégica basada en la diferenciación en las instituciones financieras; por último, el identificar las estrategias diferenciadoras más idóneas para las instituciones financieras vinculadas a la banca pública.

Desde la concepción heurística, los investigadores creen oportuno considerar lo siguiente:

- Las estrategias
diferenciadoras parten de una
necesidad de conquista del
mercado, las instituciones
financieras pretenden abrirse al
mismo; sin embargo, las
limitantes, más allá de las
normativas, se encuentran más
en la digitalización de los servicios
y la educación que requiere el
usuario para poder auto
atenderse en sus propios


requerimientos, esto último impide conectar con más posibles usuarios o clientes futuros.

- Los productos y servicios, e incluso las propias instituciones financieras, están siendo sumergidos en cambios radicales, presentándose una realidad distinta a los direccionamientos estratégicos con las cuales se han iniciado, y que aún no se adaptan; los cambios de paradigmas son muy acelerados y no hay capacidad de respuesta oportuna dentro del mismo mercado, lo cual hace que los procesos de innovación no sean trasladados de manera efectiva a los usuario o clientes.

- La identificación de estrategias innovadoras en el mercado de las instituciones financieras es un gran reto para estas mismas; hoy día el usuario - cliente está cada vez más informado sobre las propuestas, por ello, evalúa constantemente la calidad de los servicios que le prestan. El cliente mantiene un continuo repaso de los servicios, productos e infraestructura de soporte que le ofrecen o recibe a fin de valorar, desde su percepción, procesos de calidad e innovación.

Por último, se puede agregar que el conocimiento y la experiencia de los usuarios o clientes representa una novedosa dimensión en la que el mercado de las instituciones financieras debe prestar mucha atención, en la que deben conceder una prioridad máxima, dada su relevante importancia para calificar la calidad de los productos y servicios que se ofrecen o reciben.

\section{Bibliografía}

Ansoff, I. (1965). The Corporate Strategy. New York, USA: Mc Graw Hill.

Cedeño V. A; Asencio C., y Villegas A, M. (2019). Las estrategias gerenciales como base fundamental para la administración en los negocios. Universidad y Sociedad [En línea]. http://scielo.sld.cu/scielo.php ?script=sci_arttext\&pid=S221 8 $36202019000500191 \& \operatorname{lng}=\mathrm{es}$ $\& \mathrm{nrm}=\mathrm{iso}$

Chandler, A. D. (2003). Strategy and Structure. Chapters in the history of the American 
Industrial Enterprise. New York: Beard Books.

David, F. (2013). Conceptos de administración estratégica. Editorial Pearson, novena edición.

Franco, C. y Reyes, A. (2003). Los equipos de trabajo como estrategia de diferenciación. Estudios Gerenciales [En línea]

http://www.scielo.org.co/pdf/e g/v19n87/v19n87a01.pdf

Gómez, D (2019). Bueno bonito y carito. Colombia: Penguin Random House Grupo Editorial.

Guth, L. (1969). Business Policy; text and cases (Edición revisada). Illinois, USA: R. D Irwin.

Johnson, G. y Scholes, K. (1993). Exploring Corporate Strategy. Text and cases. Prentice Hall Internacional, tercera edición.

Mintzberg, H. (2013). El proceso de estrategia. Conceptos, contextos, casos. Prentice Hall Internacional.

Mintzberg, E. y Quinn, B. (2015). El proceso estratégico. Conceptos, contextos y casos. Prentice hall hispanoamericana, S.A.

Mintzberg, H. Ahlstrand, Be. Lampell J. (2016). Safari a la Estrategia. Ediciones Garnica.
Miles, R. y Snow, C. (1978). Organizational strategy, structure and process. New York. McGraw-Hill.

Narver, J. y Slater, S. (1990). The effect of a market orientation on business profitability. Journal of Marketing, vol. 54, pp. 20-35.

Porter, M. (1987). Ventaja competitiva. México. Cecsa.

Vegas, M., H. (2016). La teoría fundamentada como herramienta metodológica para el estudio de la gestión pública local. Revista Venezolana de Gerencia. [En línea] https://www.redalyc.org/jatsR epo/290/29048812004/index. html

Smith, T. (1977). Dynamic Business Strategy. The art of planning for sucess. New York, USA. McGraw Hill. 